\title{
Research on soil moisture information acquisition and monitoring system based on wireless communication
}

\author{
Tengda Qie, Liying Cao ${ }^{1}$, Guifen Chen \\ School of Information Technology, Jilin Agricultural University , Changchun 130118 \\ 694053938@qq.com
}

Key words: Internet; soil; soil moisture; real-time monitoring;

\begin{abstract}
. according to the water and fertilizer resources waste, serious shortage and low utilization rate, environmental pollution and other issues, put forward a kind of soil moisture monitoring system based on Internet of things is one of the scientific and technological content of the highest, integration of the most comprehensive management technologies for modern agriculture. Its practical application and rapid development will cause the human to fully tap the production potential of farmland, rational use of water and fertilizer resources, reduce environmental pollution and greatly improve the yield and quality of agricultural products become possible. This paper mainly for soil moisture information collection and remote transmission, based on B / S mode software were systematic design. Put forward the measures for the implementation of the software of the whole system, the soil moisture monitoring, can effective drought, pest damage early warning and provide a reliable basis.
\end{abstract}

\section{Introduction}

The agricultural production environment in China is a complex ecosystem, including soil, fertilizer, water, light, temperature, air and biological factors, environmental data on farmland for rapid and accurate acquisition, transmission, control, system analysis of direct and indirect factors, is conducive to scientific management of crops the production and application at home and abroad. From the perspective of intelligent agriculture, can realize the real-time acquisition of environmental information utilizing agriculture intelligent sensor, using self-organizing intelligent networking can be submitted to remote real-time data collection, and the biological information acquisition method is applied to the wireless sensor node, through a variety of instruments, real-time display or as automatic control variables involved in the automatic control field, and provide scientific basis for crop production and precise control, optimization of crop life The environment, not only can obtain optimal conditions for crop growth, the implementation of precision fertilization, pest warning diagnosis and treatment, also can maximally improve the practical productivity of the agriculture of our country, is an effective approach to realizing the sustainable development of agriculture of high quality, high yield, low energy consumption and environmental protection.

\section{Related technology}

A. wireless communications

With the rise of China's science and technology, mobile communication, though started late, but has come from behind, the entire land area basically covering all mobile communication signals. Mobile communication technology update replacement is like bamboo of steadily rising, the third generation, the fourth generation of mobile communication technology have in China laying and development. Although this is so, but the second generation mobile communication technology (GSM global mobile communication system) is still the mainstream of Chinese society, in the future for a long period of time, it still has an irreplaceable role.

In general, will be from the customer and the operator's point of view, GSM still has great

${ }^{1}$ Corresponding author 
vitality and advantage: because of efficient modulation, voice encoding, channel equalization optimization technology, the system has very high spectrum utilization rate; as the air interface and the digital voice transmission technology and GSM code encoding feature definition, more than one a threshold, voice quality can always at the same level, so you can get the communication signal is very stable; the GSM standard provides an open interface, the interface is not limited to the air interface, and directly on the network equipment and entities, such as the interface between $\mathrm{A}$ and Abs network interface; through the use of authentication, encryption and TMSI number, realize a security communication function, network access authentication is used to authenticate users, and the use of air interface encryption by SIM network card and AUC key Decision; usually use ISUP and top interfaces with other network connection; roam as an important feature of mobile communication, it symbolizes the customers to spontaneously from the jump to another network, global roaming, GSM system, granted requires moving some agreement exists between network operators.

B. introduction to solar power technology

Solar energy is a kind of clean and renewable resources, more and more people pay attention to, affect people's life and work widely. One of the most important way is to use solar energy through some special devices to convert into electrical energy, and solar battery is one of the way. In this paper, we will use solar panels to information acquisition terminal for power supply, wherein, the charging control module chip such as the use of CN3722 Yun Electronics production, CN3722 is to utilize the solar energy to mode PWM step-down power supply IC (integrated circuit can change with the degree of strong solar power tracking. As the information collection terminal to supply the use of solar energy, not only solves the problem of power supply in remote data collection at the same time, green environmental protection, clean and safe.

\section{The design and implementation of monitoring system}

A. system function

Based on the networking of soil moisture monitoring system by wireless GPS sensor part, wireless monitoring, remote control center, and users use the PC, web, touch screen, smart phones, micro channel platform multiple channels, acquisition land related information.

Wireless sensor nodes are deployed in the monitoring region node by self-organization composed of wireless network to realize the real time monitoring and collecting network of various monitoring object within the region of information distribution, through wireless sensor and GIS technology in black soil, chernozem, meadow soil, and soil sampling.

The use of database technology, artificial intelligence technology, data mining technology and space-time processing technology for data processing and storage, processing and analysis using expert system technology, database processing technology, cloud services technology and GIS technology, the monitoring center is composed of the server, console, visual screen server etc. monitoring software, storage servers are treated soil moisture data.

Visual screen server deployed on a central server, can collect, the processing of the collected data, if it exceeds the preset worth automatic alarm, information is sent to the terminal, the operation is simple, can query the historical data, historical data list, and curves.

B. experimental results

Temperature distribution of soil moisture during the growing period of Maize

According to the dynamic monitoring of $0-20 \mathrm{~cm}, 20-40 \mathrm{~cm}, 40-60 \mathrm{~cm}, 60-80 \mathrm{~cm}$ four levels of soil moisture, soil moisture, soil temperature data, soil moisture, temperature change trend chart as shown in figure 1- figure 4. 


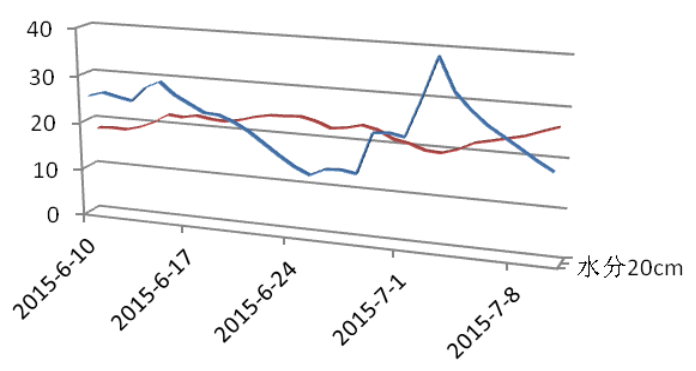

Fig. 1 change trend of soil temperature and moisture in crop growth period $0-20 \mathrm{~cm}$

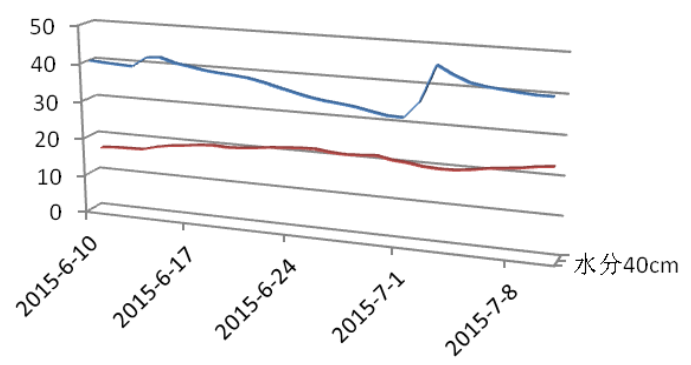

Fig. 2 change trend of soil temperature and moisture in crop growth period $20-40 \mathrm{~cm}$

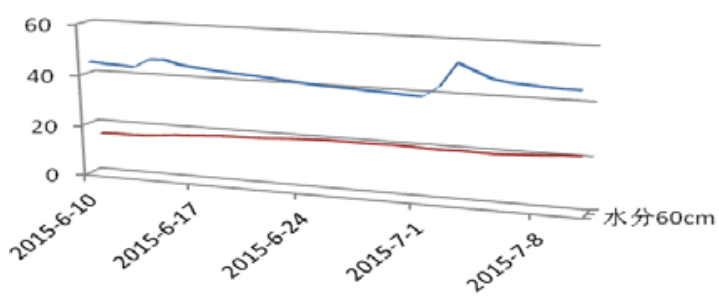

Fig. 3 change trend of soil temperature and moisture in crop growth period $40-60 \mathrm{~cm}$

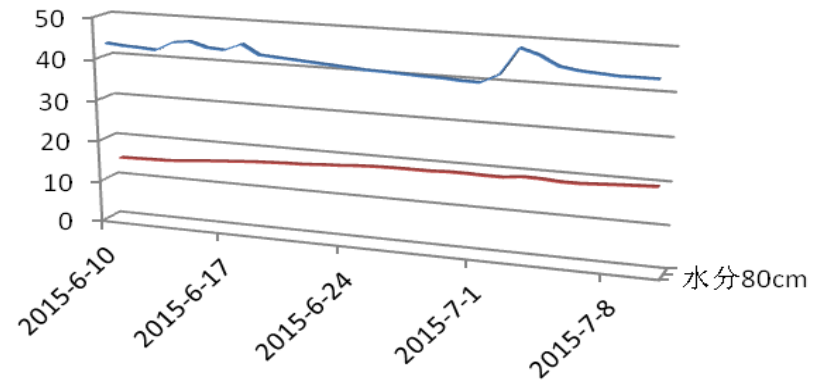

Fig. 4 change trend of soil temperature and moisture in crop growth period $60-80 \mathrm{~cm}$

From Fig. 1 - 4 can be seen in the growth period, with little change in soil temperature, and soil moisture change obvious; soil moisture in at the end of June was the lowest in July, around the four contents. Soil temperature in June around the 10th lowest in July around the 10th highest temperature;

\section{Summary}

Can use GSM short message method to achieve the results of this study demonstrated the remote collection and transmission of moisture, this system and some other such as the use of ZigBee communication system has considerable advantages compared, the future is a matter of soil moisture monitoring or atmospheric monitoring, etc. would is the mobile communication in the world. Of course because of the limited ability of the designer, system design can not be perfect and many aspects can be extended and discuss, hope with the leaping development of communication technology, later of the system were expanded and upgraded, the function will more and more powerful. 


\section{Acknowledgments}

This work was funded by The key project of the national Spark Program in 2015(2015GA660004) , Science and technology development project of Jilin Province(20160412034XH).

\section{Reference}

[1] history Guobin GPS and GIS technology in monitoring system of precision agriculture research and application progress [J]. Hubei Agricultural Sciences, 2011,10:15-19. (50): 1948-1950.

[2] Zhang Yujuan, Liu Dandan, Liu Jiang. Research on modeling methods of urban terrain: a case study of Heilongjiang Academy of Engineering $[\mathrm{J}]$ forestry science and technology information, 2013,45 (3): 60-62.

[3] Zhang Haoran. GIS technology [J]. surveying and spatial information in mobile domain, 2014,37 (4): 137-138141.

[4] Cheng Penggen, Liu Shaohua, Wang Wei, Chen Honghua. Research and application of 3D geological model construction method [J]. Journal of Jilin University: Earth Science Edition, 2004,4 (2): 309-313.

[5] Ying Biao Chen, Zhong Ershun. Geological exploration of mine 3D visualization management system and modeling [J]. Mining research and development, 2004,24 (1): 37-40.

[6] Wang Minghua, white clouds. The 3D geological modeling research on the current situation and development trend of [J]. soil engineering and foundation, 2006,20 (4): 68-70.

[7] Zhang Kaixuan, Wu Wenbo, Bai Yunfeng, et al. 3D visualization of geological ArcGIS [J]. Journal of Liaoning Technical University based on 2007,26 (3): 345-347. 